Cahiers $d u$ MONDE RUSSE

\section{Cahiers du monde russe}

Russie - Empire russe - Union soviétique et États indépendants

$50 / 4 \mid 2009$

Varia

\title{
Alexander S. Morrison, Russian Rule in Samarkand 1868-1910
}

Isabelle Ohayon

\section{OpenEdition \\ Journals}

Édition électronique

URL : https://journals.openedition.org/monderusse/7220

DOI : $10.4000 /$ monderusse. 7220

ISSN : $1777-5388$

Éditeur

Éditions de l'EHESS

\section{Édition imprimée}

Date de publication : 15 décembre 2009

Pagination : 890-892

ISBN : 978-2-7132-2261-0

ISSN : $1252-6576$

Référence électronique

Isabelle Ohayon, «Alexander S. Morrison, Russian Rule in Samarkand 1868-1910», Cahiers du monde russe [En ligne], 50/4 | 2009, mis en ligne le 12 janvier 2011, consulté le 03 septembre 2022. URL :

http://journals.openedition.org/monderusse/7220; DOI : https://doi.org/10.4000/monderusse.7220

Ce document a été généré automatiquement le 3 septembre 2022

Tous droits réservés 


\title{
Alexander S. Morrison, Russian Rule in Samarkand 1868-1910
}

\author{
Isabelle Ohayon
}

\section{RÉFÉRENCE}

Alexander S. MORRISON, Russian Rule in Samarkand 1868-1910. A Comparison with

British India. Oxford - New York : Oxford University Press, 2008, 364 p.

1 Avec Russian Rule in Samarkand: A Comparison with British India, Alexander Morrison réinscrit l'histoire de la colonisation de l'Asie centrale et de l'Empire russe dans le temps $\mathrm{du} \mathrm{XIX}^{\mathrm{e}}$ siècle européen et des grands empires coloniaux, le cas russe - et plus particulièrement l'expérience centrasiatique - ayant résisté jusqu'à ces dernières années à la comparaison. Disons-le d'emblée : si le sous-titre de l'ouvrage de Morrison affiche une perspective comparatiste avec l'Inde britannique, il ne propose pas en réalité une comparaison rigoureuse terme à terme pour différentes raisons légitimes que l'auteur explicite largement en introduction. En effet, le grand nombre de dissemblances entre les deux Empires, russe et britannique - ceux-ci eussent-ils été fascinés l'un par l'autre en leur temps - rend l'exercice malaisé. La conquête du Turkestan commence en 1865 alors que la domination britannique en Inde se consolide à la fin du XVIII siècle après plusieurs décennies de suprématie commerciale et politique de la Compagnie britannique des Indes orientales. La première est motivée par des considérations exclusivement militaires sur une terre lointaine et peu accessible dont l'occupation demeure longtemps coûteuse et déficitaire, tandis que la colonisation de l'Inde poursuit des ambitions économiques qui dépassent les intérêts militaires de la couronne britannique sur un territoire bien plus vaste dont la proximité avec le centre du pouvoir impérial est renforcée grâce à l'ouverture du canal de Suez. Les uns ont affaire à un territoire "vide" - la population du Turkestan s'élève à moins de huit millions d'habitants en 1897, tandis que les autres doivent administrer un monde " plein ", l'Inde comprenant près de 300 millions d'habitants en 1900. Enfin - et Alexander Morrison ne cessera d'insister sur ce point -, le Turkestan bénéficie d'une certaine autonomie dans 
son mode de gouvernement, alors que le contrôle exercé par les administrateurs de l'Inde, comme les relations avec la métropole, sont bien plus resserrés. Néanmoins, l'auteur affirme qu'une tentative de comparaison est à même de rendre compte de la diversité des réponses apportées par les pouvoirs coloniaux à des problèmes communs et de distinguer ce qui relève des spécificités de la colonisation russe de ce qui procède de logiques impériales européennes.

Quoi qu'il en soit, les deux expériences coloniales n'ont pas le même statut scientifique dans ce travail, la colonisation russe en Asie centrale étant bien plus approfondie que la britannique, laquelle est d'autre part fort mieux documentée sur le plan historiographique. Par le choix même de la région choisie par Alexander Morrison - Samarkand et la vallée du Zeravšan, qui constitue un territoire administratif du Turkestan -, Russian rule in Samarkand s'apparente davantage à une monographie. Celle-ci résulte en effet d'un minutieux travail dans les archives de Russie (GARF, RGIA, RGVIA, Archives de l'Institut d'orientalisme de Saint-Pétersbourg) et d'Ouzbékistan (CGARUz), d'une part, et plus à la marge dans celles de New Delhi (Archives nationales de l'Inde) et des collections orientales de la British Library, d'autre part. L'ouvrage, tiré de la thèse de l'auteur, comprend sept parties respectivement consacrées : au contexte général de la colonisation du Turkestan (I), à la religion et au rapport à l'islam (II), à la création d'une administration locale (III), à la bureaucratie militaire (IV), à l'administration autochtone à Samarkand (V), à l'irrigation (VI), au système judiciaire et aux qazis ou juges « chariatiques» (VII).

3 Centré sur les instruments régaliens de l'État russe dans la colonie turkestanaise, qu'il s'agisse des autorités impériales, de leurs intermédiaires autochtones, du système fiscal ou de la justice, non seulement ce travail renouvelle une historiographie très pauvre qui se limitait à la littérature soviétique locale, mais il contribue également à des apports importants concernant le processus même de construction de l'État colonial à l'échelle d'une région. Faisant écho à l'ouvrage de Jeff Sahadeo, Russian Colonial Society in Tashkent, il dresse le tableau d'un système administratif à la fois autonome et déficient, animé par un monde de fonctionnaires ignorants non initiés aux langues locales, souspayés, surmenés, et qui sont "naturellement » sujets à la corruption. Dépendant des élites autochtones, ces fonctionnaires utilisent leur autorité pour asseoir leur pouvoir auprès de ces dernières, qui se constitueront rapidement en clientèles attitrées.

Pourtant, Morrison souligne que l'ambition ultime de la colonisation du Turkestan demeure, selon les discours et les choix théoriques de l'État, le rapprochement socioculturel des musulmans et des Russes, objectivé par le concept de sbliženie. Mais ce sont justement le faible niveau de formation des administrateurs, ainsi que leurs difficultés quotidiennes dues notamment au manque de moyens financiers, qui sont en grande partie à l'origine d'une mise à l'écart de cet idéal assimilationniste au profit d'une indifférence envers les populations et du dessein colonial, attitude que l'auteur lui-même qualifie de "négligence accidentellement bienveillante"(p.291). Même sur le plan économique, l'exploitation du coton et la modernisation des infrastructures d'irrigation restent très modestes, bien qu'elles produisent un impact politique fort auprès des populations, puisqu'elles se trouvent au cœur des relations entre colons et médiateurs autochtones. Ces derniers, les chefs de village en particulier, détiennent en effet un savoir technique précieux qui les place en position avantageuse face au colonisateur, ce dont ils ne manquent pas de tirer profit en termes d'autorité sociale (voir les chapitres consacrés respectivement à l'irrigation et à la justice). La colonisation, comme toujours, contribue 
de ce point de vue à redéfinir les hiérarchies de la société en place. Finalement l'inefficacité, voire l'inertie, des représentants de l'Empire, qui contraste infiniment avec la situation en Inde britannique, laisse davantage place à une crainte du fanatisme religieux et des soulèvements; et, en dépit de la méfiance de rigueur envers l'islam, cette attitude explique une politique qualifiée par le gouverneur général du Turkestan de 1867 à 1882, Von Kaufman, d'ignorirovanie (ignorance, méconnaissance). Celle-ci consiste à ne pas prêter une attention particulière à l'islam et à peu intervenir dans la gestion de ses institutions, ce qui se traduit notamment par le refus de rattacher les musulmans du Turkestan au muftiat d'Orenburg par peur d'une mainmise des élites tatares et bachkires sur leurs confrères d'Asie centrale. En même temps, cette politique exclut toute conversion à l'orthodoxie.

5 Ainsi, c'est un tableau assez édulcoré de la domination coloniale que nous livre Alexander Morrison dans son ouvrage. Du fait des sources utilisées, qui émanent toutes de l'administration tsariste, il ne rend compte, en effet, que du regard du colonisateur ; et bien qu'il utilise largement les pétitions adressées aux administrateurs tsaristes par les villageois turkestanais dénonçant la corruption des médiateurs autochtones, il n'exploite pas tout leur potentiel. En les prenant pour preuve d'une corruption rampante, certainement existante, d'une part il néglige d'y voir un moyen de manipuler le pouvoir colonial dans le but de régler des comptes, des conflits ne relevant pas de la juridiction impériale, ou de l'instrumentaliser dans le cadre de rivalités entre élites locales - phénomène que l'on retrouve couramment dans les premières décennies du régime soviétique en Asie centrale. D'autre part, il se prive d'une discussion sur le rôle et le statut de la corruption des autochtones comme forme de déloyauté, voire de résistance, au pouvoir colonial - et par conséquent sur la relation entre intermédiaires et administrateurs. Ceci étant, bien qu'il ait tendance à minorer l'impact de la colonisation sur les sociétés centrasiatiques ainsi que la violence culturelle et symbolique que celle-ci génère, cet ouvrage constitue, par sa rigueur, son degré d'érudition et sa nouveauté, une contribution de grande qualité à l'histoire impériale centrasiatique en essor ces dernières années. 